\title{
Review Article \\ Potential Role of Protein Disulfide Isomerase in Metabolic Syndrome-Derived Platelet Hyperactivity
}

\author{
Renato Simões Gaspar, ${ }^{1}$ Andrés Trostchansky, ${ }^{2,3}$ and Antonio Marcus de Andrade Paes ${ }^{1,3}$ \\ ${ }^{1}$ Laboratory of Experimental Physiology, Department of Physiological Sciences, Federal University of Maranhão, São Luís, MA, Brazil \\ ${ }^{2}$ Departamento de Bioquímica and Center for Free Radical and Biomedical Research, Facultad de Medicina, \\ Universidad de la República, Montevideo, Uruguay \\ ${ }^{3}$ Health Sciences Graduate Program, Biological and Health Sciences Center, Federal University of Maranhão, São Luís, MA, Brazil
}

Correspondence should be addressed to Antonio Marcus de Andrade Paes; marcuspaes@ufma.br

Received 12 August 2016; Revised 17 October 2016; Accepted 1 November 2016

Academic Editor: Nageswara Madamanchi

Copyright (C) 2016 Renato Simões Gaspar et al. This is an open access article distributed under the Creative Commons Attribution License, which permits unrestricted use, distribution, and reproduction in any medium, provided the original work is properly cited.

\begin{abstract}
Metabolic Syndrome (MetS) has become a worldwide epidemic, alongside with a high socioeconomic cost, and its diagnostic criteria must include at least three out of the five features: visceral obesity, hypertension, dyslipidemia, insulin resistance, and high fasting glucose levels. MetS shows an increased oxidative stress associated with platelet hyperactivation, an essential component for thrombus formation and ischemic events in MetS patients. Platelet aggregation is governed by the peroxide tone and the activity of Protein Disulfide Isomerase (PDI) at the cell membrane. PDI redox active sites present active cysteine residues that can be susceptible to changes in plasma oxidative state, as observed in MetS. However, there is a lack of knowledge about the relationship between PDI and platelet hyperactivation under MetS and its metabolic features, in spite of PDI being a mediator of important pathways implicated in MetS-induced platelet hyperactivation, such as insulin resistance and nitric oxide dysfunction. Thus, the aim of this review is to analyze data available in the literature as an attempt to support a possible role for PDI in MetS-induced platelet hyperactivation.
\end{abstract}

\section{Introduction}

Definition of Metabolic Syndrome (MetS) has been a matter of intense scientific output over the last decades, reaching a consensus by The National Cholesterol Education ProgramAdult Treatment Panel (NCEP ATP III) to include five major features: visceral obesity, hypertension, dyslipidemia, insulin resistance, and high fasting glucose levels. Diagnostic criteria for MetS must include at least three out of these five features [1]. MetS has become a worldwide epidemic, alongside with a high socioeconomic cost whose prevalence widely ranges from $8 \%$ to $43 \%$ in men and $7 \%$ to $56 \%$ in women [24]. Importantly, the presence of MetS is associated with a substantial 5-fold increased risk of developing diabetes mellitus (DM) and a 2-fold increase in the development of cardiovascular disease, concurring for higher likelihood to suffer ischemic events [2-5]. In fact, MetS is an independent risk factor for cardiovascular disease (CVD), leading patients to exhibit a prothrombotic and proinflammatory status $[6,7]$. As a long-term outcome, MetS individuals tend to develop atherosclerotic plaque as a chronic inflammatory process characterized by increased levels of inflammatory markers, such as tumor necrosis factor $\alpha$, interleukin-6, leptin, angiotensin II, and plasminogen activator factor 1 , all of them capital prothrombotic factors $[6,7]$.

Besides increased inflammatory markers, the prothrombotic state in MetS is mainly caused by endothelial dysfunction and platelet hyperactivity. Both lipotoxicity and insulin resistance contribute to increased oxidative stress (OxS) in the endothelium, leading to enhanced production of reactive oxygen species (ROS) by various isoforms of NADPH oxidase (Nox) and reduced nitric oxide (NO) production and bioavailability, consequent to lower expression and/or uncoupling of endothelial nitric oxide synthase (eNOS) as well as increased reactivity with superoxide $\left(\mathrm{O}_{2}{ }^{-}\right)$[8]. Platelets are key players involved in pathologic thrombosis through 
increased adhesion to the compromised endothelium, being also affected by the increased OxS present in MetS $[9,10]$. It has been shown that MetS subjects have increased mean platelet volume, an independent predictor of vascular events [11]. Moreover, there is an increase of proaggregatory and prothrombotic mediators such as thromboxane $\mathrm{A}_{2}\left(\mathrm{TxA}_{2}\right)$ and adhesion molecules such as P-selectin, while inhibitory components, like NO, are decreased [12]. Overall, there is an increase in prothrombotic factors with a concomitant decrease in inhibitory components in both endothelium and platelets that concur for increased CVD in MetS.

The Protein Disulfide Isomerase (PDI) is a family of thiol isomerases originally found in the endoplasmic reticulum (ER) that were later discovered in the cytosol and surface of endothelial cells and platelets, among others $[13,14]$. The most abundant and physiologically relevant member is PDIA1, the product of the $P 4 H B$ gene, with a molecular weight of $57,000 \mathrm{Da}$ and five subunits: four thioredoxin-like domains $\left(\mathrm{a}-\mathrm{b}-\mathrm{b}^{\prime}-\mathrm{a}^{\prime}\right)$, one C-terminal extension domain, besides one $\mathrm{x}$ linker sequence between $b^{\prime}$ and $a^{\prime}[15,16]$. PDIA1 is also an important regulator of thrombus formation, rapidly binding to $\beta_{3}$ integrins on the endothelium upon injury [17]. In platelets, membrane PDI members, such as PDIA1, ERP5, and ERP57, are known for their paramount importance in platelet aggregation through the isomerization of a disulfide bond in the $\alpha_{2 \mathrm{~b}} \beta_{3}$ integrin, which is the final convergent pathway in virtually all mechanisms of platelet aggregation [18]. In addition, platelet surface PDI participates in platelet adhesion through a close interaction with collagen receptor $\alpha_{2} \beta_{1}$ [19], GP1b $\alpha$ [20], vitronectin [21], and thrombospondin 1 [22]. Despite the already established importance of PDI proteins, precise mechanisms through which surface thiol isomerases interact with integrins and other platelet membrane receptors are still unclear. Since MetS involves many risk factors associated to changes in the coagulation pathway, the aim of this review is to analyze the potential role of platelet surface PDIA1, henceforth referred as PDI, as a central player in platelet hyperactivation under MetS.

\section{Metabolic Syndrome and Vascular Oxidative Stress}

ROS, specially $\mathrm{O}_{2}{ }^{--}$and hydrogen peroxide $\left(\mathrm{H}_{2} \mathrm{O}_{2}\right)$, are ubiquitous oxidants of moderate reactivity and brief halflife found in virtually all biological systems as byproducts of oxygen metabolism [23]. At low levels, ROS are key players in many biochemical processes, such as signaling cascades, gene transcription, cellular growth and migration, and apoptosis [24]. In particular at the vascular system, ROS participate in controlling vasodilation and platelet adherence/aggregation [23]. However, when ROS generation is excessive and not compartmentalized, exceeding endogenous antioxidant capacity, cells and tissues progress to OxS, which is considered an early event in the pathophysiology of most chronic noncommunicable diseases associated to MetS [25, 26].

The vascular OxS observed in MetS leads to a change in plasma redox state, inciting a prooxidant environment due to the imbalance of two central thiol/disulfide couples, glutathione/glutathione disulfide (GSH/GSSG), and cysteine/cystine (Cys/CySS) [25, 27]. The reduced partners (GSH or Cys) help in maintaining the thiol/disulfide redox state in proteins, as well as the redox state of ascorbate and vitamin $\mathrm{E}$ in their reduced healthy forms by their participation in peroxides removal. Under prooxidant conditions, GSH levels decline in both intracellular and extracellular environment of vascular cells in parallel with an increase in GSSG. Thus, measurement of reduced and oxidized products, as well as their ratios, can provide a useful indicator of $\mathrm{OxS}$ in human plasma [25]. Several thiol-containing proteins at the surface of vascular cells, such as thioredoxin and its relatives from PDI family, in response to variable concentrations of ROS, alter the redox state of critical thiols that leads to ROSdriven cellular activation [28]. Noteworthy, recent reports have corroborated the impact of MetS on plasma redox state, with particular emphasis on the assessment of redox status as a tool to predict different outcomes in prediabetic patients $[29,30]$.

The phagocytic and nonphagocytic isoforms of Noxes are the primary source of ROS and have been consistently implicated in different vascular pathologies [31, 32]. Nox complexes are composed of multiple subunits comprising catalytic (Nox 1-5) and regulatory (p22 $2^{\text {phox }}, \mathrm{p} 40^{\text {phox }}, \mathrm{p} 47^{\text {phox }}$, p6 $7^{\text {phox }}$, Noxo 1, Noxa 1, and the small GTPases Rac 1 and Rac 2) components, whose expression may vary according to the cell type [33]. Nox4 is associated to cell differentiation of vascular smooth muscle cells [34], whereas Noxl supports cellular proliferation and migration [35]. Endothelial cells express four Nox isoforms (Nox1, Nox2, Nox4, and Nox5), from which Nox 4 is the most highly expressed, and promote $\mathrm{H}_{2} \mathrm{O}_{2}$-derived endothelium preservative actions [36, 37]. On the other hand, expression levels of the other isoforms have been directly implicated in endothelial dysfunction [38].

In platelets, Nox 2 was identified by the localization of membrane $\mathrm{p} 22^{\text {phox }}$, cytosolic $\mathrm{p} 47^{\text {phox }}$ subunits, and more recently the catalytic gp91 ${ }^{\text {phox }}$ subunit $[39,40]$. Similarly, Noxl is also expressed in human platelets, although in a lesser extent when compared to Nox2 [41]. The same study failed to localize Nox4 and Nox5 in platelets, even though further studies are needed to address this matter. Since platelets express Nox1 and Nox2, Delaney and colleagues [42] compared the differential roles of these enzymes in platelet activation and thrombosis. They showed that Nox2, but not Noxl, is required for thrombus formation, whereas none of the enzymes altered tail bleeding time in mice, suggesting further studies should focus on whether Nox-dependent ROS generation may become a potential antithrombotic target without significant bleeding complications [42].

In addition to Nox enzymes, there are different nonenzymatic and enzymatic pathways involved in the formation of ROS in vascular milieu, among them, spontaneous dysmutation of oxygen, leakage of the mitochondrial electron transport chain, myeloperoxidase, xanthine oxidase, cyclooxygenases, and uncoupled NOS [43]. Virtually all these mechanisms may concur for MetS-associated cellular damage, which leads to increased formation of advanced glycation 
end products (AGE) and its receptors, hexosamine pathway overactivity, increased polyol pathway flux, activation of protein kinase C isoforms [44, 45], lipotoxicity [46], and increased inflammatory profile [47]. Therefore, endothelial cells and platelets from MetS patients suffer from this marked increase in ROS generation, playing a pivotal role in the macro- and microvascular complications of this syndrome.

\section{Metabolic Syndrome and Platelet Hyperactivity}

The damage caused by $\mathrm{OxS}$ has been shown to increase platelet aggregation in MetS subjects and decrease aspirin response in DM $[6,10,48]$. These can be explained by several mechanisms: increased platelet secretion of $\mathrm{TxA}_{2}$ and prostaglandins (PG), decreased expression of NOS in both endothelium and platelets in addition to a decreased production of prostacyclin $\left(\mathrm{PGI}_{2}\right)$ at the endothelium; decreased platelet response to $\mathrm{NO}$ and platelet insulin resistance. Among these, the development of insulin resistance and the impairment of NO homeostasis are arguably the most substantial pathways involved in platelet hyperactivation in MetS.

\subsection{Thromboxane and F2-Isoprostanes Overproduction in} Platelets. $\mathrm{TxA}_{2}$ is one of the byproducts of arachidonic acid (AA) oxidation by prostaglandin endoperoxide $\mathrm{H}_{2}$ synthase1 (PGHS-1), also known as cyclooxygenase-1 (COX-1), in platelets [49]. $\mathrm{TxA}_{2}$ is synthesized by platelets and acts as an agonist in platelet aggregation and activation, through the ligation of its own $G$ protein-coupled receptor, leading to increased $\alpha_{2 \mathrm{~b}} \beta_{3}$ expression, the latter being blocked by the COX-inhibitor aspirin [50]. Besides $\mathrm{TxA}_{2}, \mathrm{~F} 2$-isoprostanes are also derived from AA oxidation, stimulating platelet aggregation and complementing $\mathrm{TxA}_{2}$ actions. Specifically, 8iso-PGF $2 \alpha$ is secreted by platelets upon stimulus, enhancing platelet activation and adhesive reactions to other agonists at low concentrations, through interaction with thromboxane receptor $[51,52]$. Noteworthy, urinary secretion of 8-isoPGF2 $\alpha$ is also considered a clinical marker of platelet activity [52], which has been found to be increased in obese women [53].

Increased platelet ROS formation in MetS overactivates platelet Nox 2 partly through oxidized low-density lipoprotein (oxLDL) ligation of platelet CD36 [54], causing an increase in cytosolic peroxide tone, that is, increased peroxynitrite generation, that subsequently stimulates COX-1 activity $[49,55]$. This setting enhances $\mathrm{TxA}_{2}$ and 8-iso-PGF2 $\alpha$ levels through lipid peroxidation and redox-catalyzed conversion of AA into F2-isoprostanes [52]. Interestingly, it seems platelet Nox2 is an important regulator of 8 -iso-PGF $2 \alpha$, since chemical or hereditary inhibition of Nox2 strongly decreases 8-isoPGF2 $\alpha$ generation in platelets [39]. Since COX-1 activity is based in the continuous generation of a lipid-derived radical, besides the reductant environment of the platelet, $\mathrm{TxA}_{2}$ pathway is continuously interrupted and requires a permanent reactivation by peroxides [49]. Therefore, OxS can exacerbate platelet aggregation in MetS by changing the intracellular peroxide and peroxynitrite levels, culminating in $\mathrm{TxA}_{2}$ and F2-isoprostanes overproduction, a mechanism at least partially regulated by Nox 2 .

3.2. Dysfunctional NO Effects in Platelets. NO is a potent vasodilator and antiplatelet mediator whose bioavailability is inversely correlated with cardiovascular risk [56-59]. Under normal conditions, NO derived from endothelial and platelet NOS diffuses toward circulating platelets in order to activate guanylate cyclase (GC), thus augmenting cyclic guanosine monophosphate (cGMP) levels. Increased levels of cGMP as well as cyclic adenosine monophosphate (cAMP) induce the phosphorylation of vasodilator-stimulated phosphoprotein (VASP) that will inactivate integrin $\alpha_{2 \mathrm{~b}} \beta_{3}$ [60-62]. NO also decreases intracellular $\mathrm{Ca}^{2+}$ levels [63], inhibits thromboxane receptors in platelets [64], and diminishes platelet recruitment in thrombus formation [65]. Furthermore, in vascular smooth muscle cells, NO is a potent vasodilator that reduces intracellular $\mathrm{Ca}^{2+}$ levels by the abovementioned mechanisms [66].

However, in the context of increased OxS induced by MetS or aging, intraplatelet ROS overproduction decreases NO bioavailability by forming reactive nitrogen species (RNS), such as peroxynitrite, leading to platelet hyperactivation [61, 67]. Of note, peroxynitrite induces platelet aggregation with increased intracellular $\mathrm{Ca}^{2+}$ concentration [68], while it also oxidizes several proteins that blunt NOS and reduce platelet antioxidant capacity [58,69]. Platelet NOS was found to be downregulated in MetS patients, which could partially explain the decreased NO production in these subjects when compared to healthy ones [70]. Besides compromised NO bioavailability, platelets from patients with unstable coronary syndrome showed impaired antiplatelet response to the NO donor sodium nitroprusside, suggesting a platelet NO resistance that could be associated to increased OxS [71]. Thus, OxS causes not only a decrease in platelet NO bioavailability, but also a dysfunctional response to its action.

3.3. Dysfunctional Insulin Effects in Platelets. Since the discovery of insulin receptors in human platelets, insulin signaling has been considered an important regulator of its function. Hajek and colleagues were the first to demonstrate that platelets possess insulin receptors, with a density of roughly 500 receptors/cell, comparable to other insulinsensitive cell types [72]. Physiologically, insulin binds to its membrane receptor, provoking the autophosphorylation of its $\beta$-chain and activating the classical insulin's signaling pathway [73]. In fact, it has been shown that insulin inhibits platelet aggregation in healthy nonobese subjects [74, 75], by a mechanism involving inhibition of tissue factor (TF) and modulation of plasminogen activator inhibitor-1 (PAI1) concentrations [69]. Moreover, other groups reported that insulin decreases intraplatelet $\mathrm{Ca}^{2+}$ content [76] and reduces platelets' response to agonists possibly due to the activation of eNOS [77] and sensitization of platelets to the inhibitory effects of NO $[12,78]$.

Similar to NO dysfunctional effects in MetS, it has been shown that obese DM subjects blunted insulin's antiplatelet 
Normal condition

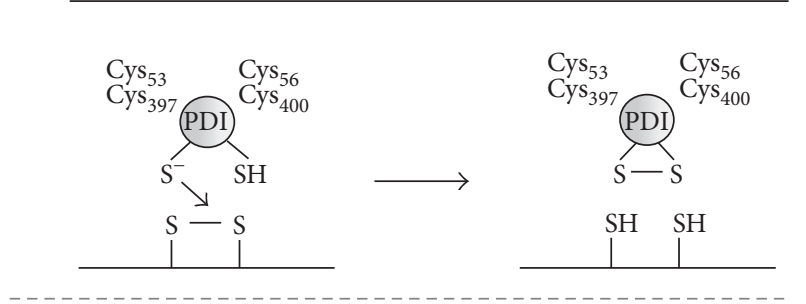

Oxidative stress

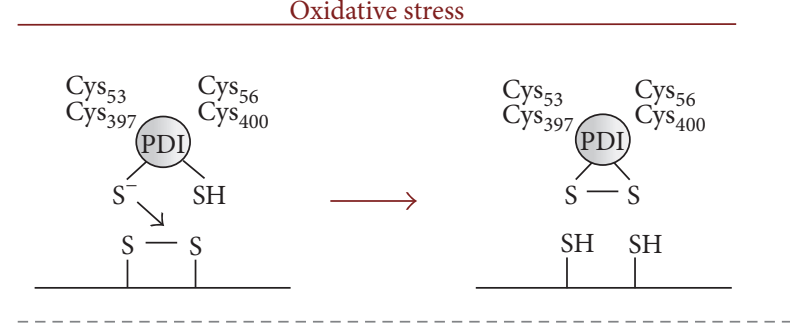

(a) Reduction
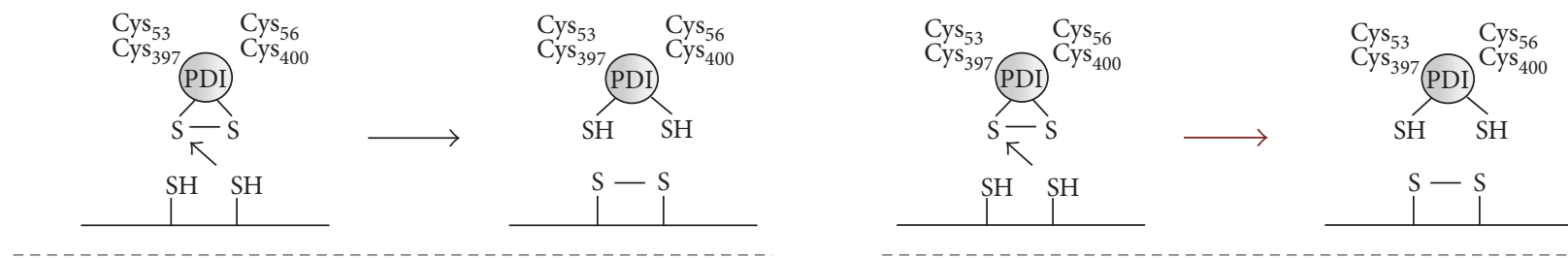

(b) Oxidation
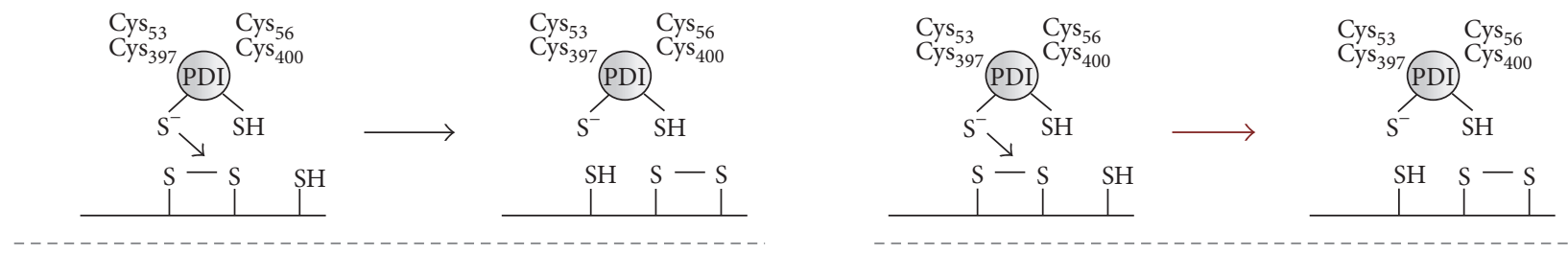

(c) Isomerization
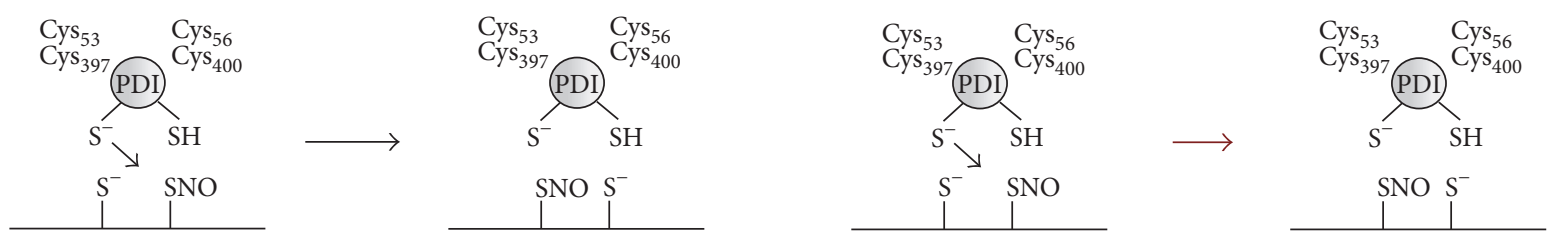

(d) NO transfer

FIGURE 1: Reactions catalyzed by PDI and possible unbalance in oxidative stress. In (a), PDI catalyzes reduction of a disulfide bond to a dithiol through an attack from its thiolate anion located in $\mathrm{Cys}_{53}$ or $\mathrm{Cys}_{397}$, whereas in (b) shows PDI oxidizing a dithiol into a disulfide bond. In (c), PDI isomerizes a disulfide bond in the same molecule. These reactions are expected to be increased in OxS, since the reduction of disulfide bonds has been shown to promote platelet aggregation and isomerization is an essential step towards $\alpha_{2 b} \beta_{3}$ activation [18]. In (d), PDI reacts with NO to promote transnitrosation, shifting NO from one molecule to another or within the same molecule. It should be noted that PDI might also catalyze denitrosation, releasing NO from S-nitrosothiols. This reaction is expected to be decreased in oxidative stress mainly due to the decreased NO bioavailability.

effects, confirming that human platelets can undergo insulin resistance. Insulin resistance is defined by the lack of insulin's actions in platelets, which downregulates IRS-1/Akt pathway, culminating in elevated intracellular $\mathrm{Ca}^{2+}$ content and proaggregatory mediators. In fact, platelets from diabetic patients exhibit faster and higher aggregation when compared to healthy ones $[69,79]$. Moreover, insulin resistance augments intraplatelet synthesis of PAI-1 and secretion of thromboxane metabolites, thus creating a proaggregatory environment [69]. Finally, there is increased thrombin and fibrin generation, with a prothrombotic fibrin clot phenotype in diabetic patients [80]. Thus, platelet insulin resistance is one of the main contributors to $\mathrm{OxS}$-derived platelet hyperactivation in MetS, even though there is no pathophysiological model to explain how platelets become insulin resistant.

\section{Protein Disulfide Isomerase and Platelet Hyperactivation in MetS}

PDI is an ubiquitous chaperone, structurally divided in five subunits: four thioredoxin-like domains $\left(a-b-b^{\prime}-a^{\prime}\right)$ and one $\mathrm{C}$-terminal extension domain, besides one $\mathrm{x}$-linker sequence between $b^{\prime}$ and $a^{\prime}[15,16]$. Among these, its catalytic redox motif CGHC is present in both a and $\mathrm{a}^{\prime}$ domains in a constant balance between disulfide and dithiol forms. These CGHC motives confer PDI its ability to catalyze oxidation, reduction, and isomerization reactions (Figure 1), through redox exchanges apparently guided by a trial and error process [16]. Even though containing a C-terminal KDEL ER-retention sequence, PDI is also found in cytosol and surface membrane of numerous cell types, including platelets 
[14]. Interestingly, besides surface membrane, platelet PDI was localized in the sarco-/endoplasmic reticulum, being mobilized to the surface during platelet activation through a mechanism requiring actin polymerization [81]. Important to this review, we refer specifically to platelet surface PDI.

In platelets, PDI is known for its paramount importance in platelet aggregation through the isomerization of a disulfide bond in the $\alpha_{2 b} \beta_{3}$ integrin [18]. Such integrin is considered the most important component and final convergent pathway in virtually all mechanisms of platelet aggregation [18]. In fact, anti-PDI antibody inhibits platelet aggregation [82], whereas the addition of reduced PDI prior to agonists enhances maximum aggregation [83]. Of note, it has been recently showed that the C-terminal CGHC motif of PDI is essential for its function in thrombus formation and platelet aggregation [83]. Moreover, PDI is also implicated in the function of other integrins, such as the collagen receptor $\alpha_{2} \beta_{1}[19]$ and the von Willebrand factor receptor glycoprotein $1 \mathrm{~b} \alpha$ [20], even though the precise mechanism of such interactions is unclear. Overall, PDI is considered a prothrombotic enzyme, directly implicated in platelet activation, aggregation, and adhesion.

Strikingly, PDI seems to be related to platelet insulin resistance and consequent hyperactivity in OxS induced by MetS. Contrasting with insulin's TF inhibition, PDI has been described as an essential component of TF activation. A proposed working model states that reduced PDI, secreted by activated platelets, reacts with low procoagulant activity TF to yield a TF with high procoagulant activity through the formation of a disulfide bond between $\mathrm{Cys}_{186}$ and $\mathrm{Cys}_{209}$ residues on TF molecule. Additionally, PDI may promote fibrin generation [84]. Overall, this suggests that while insulin inhibits TF activation, PDI works on the opposite side by augmenting TF procoagulant activity and increasing fibrin generation upon injury. Nonetheless, insulin resistance and PDI seem to exert similar effects on platelet activation. Even though no study has ever demonstrated whether PDI can desensitize platelet's insulin receptors, the likewise effects of insulin resistance and PDI on platelet function could lead to a possible connection between these two factors.

A plausible hypothesis is that PDI's procoagulant reactions could be increased in insulin resistance and OxS in detriment of decreased insulin activity or even that insulin resistance could be, at least in part, accounted for increased PDI activity. This is supported by the well-characterized in vitro reaction between PDI and insulin, where the first reduces a disulfide bond in the latter, causing the precipitation of insulin's $\beta$-chain [85]. In vivo, it has been reported that DM patients release more platelet-derived microparticles (pMPs) [86]. Importantly, pMPs contain catalytically active PDI, and DM subjects have increased levels of PDI-containing pMPs [87]. In fact, plasma samples from DM patients present roughly $50 \%$ more pMPs than healthy subjects, also exhibiting 60\% more PDI and 70\% more PDI activity [87]. Noteworthy, these microparticles were able to catalyze insulin disulfide reduction, abrogating insulin's activity, as shown by loss of Akt phosphorylation in 3T3-L1 cells [87]. Therefore, it is reasonable to suggest that the increased PDI secretion from DM platelets reduces insulin's bioavailability, contributing to the lack of insulin's action found in MetS platelets (Figure 2). Nonetheless, it should be stressed that further studies are needed to address whether PDI is an important cue in MetSinduced platelet hyperactivity, specifically if secreted platelet $\mathrm{PDI}$ is able to desensitize insulin receptor in various cell types.

PDI is also involved in platelet NO homeostasis [88], providing evidence for a paradoxical effect of PDI in platelet activation. Previous studies have shown that PDI acts as an NO carrier through vascular cells by transnitrosation reactions, exchanging the nitrosonium ions between cysteines (Figure 1(d)) [89, 90]. Likewise, it was also shown that platelet PDI denitrosates S-nitrosothiols (RSNOs), thus releasing NO and increasing its bioavailability [91]. Moreover, RSNOs seem to be denitrosated by the same CGHC active site that gives PDI its proaggregatory properties $[83,91]$. These findings were further supported by Bell et al. [92] that showed PDI is implicated in a wide range of NO-related signals and not only with RSNOs, as previously thought. However, NO can also attack PDI in an S-nitrosylation reaction, which transfers NO to critical cysteines in CGHC active sites. Such reaction inhibits PDI isomerization and chaperone activities by roughly $50 \%$, which could in turn compromise the aforementioned mechanisms of platelet aggregation through $\alpha_{2 b} \beta_{3}$ [93]. Despite acting as a NO donor, PDI paradoxically inhibits NO effects in vascular smooth cells by a thioldisulfide exchange between PDI's CGHC active site and the $\alpha$ or $\beta$ domains of soluble GC $[94,95]$. Therefore, platelet PDI improves NO bioavailability, acts as an NO carrier, while it can also be inhibited by NO itself, whereas in vascular smooth cells PDI abrogates NO effects.

Nevertheless, it is important to notice that the abovementioned studies took place under physiological conditions and the interaction between PDI and NO was not tested under increased OxS, nor was it tested in MetS subjects. One could hypothesize that increased ROS production, causing cellular damage and increased OxS, coupled with alterations in plasma GSH/GSSG and Cys/CySS could interfere with PDI's denitrosation activity or even revert its effect. Additionally, decreased NO bioavailability due to peroxynitrite formation leads to lower extents of PDI reacting with available NO and/or less NO inhibiting PDI isomerase activity, which would shift the enzyme's activity to proaggregatory pathways (Figure 2). These hypotheses are based on the well-established decrease of NO bioavailability in MetS (detailed in Section 3.2) and should be addressed in future studies, since dysfunctional NO effect on platelets is an important cue to better understand platelet hyperactivity. Nonetheless, studies are needed to investigate whether there is a link between PDI and decreased NO bioavailability or diminished platelet NO response, given that this protein is of capital importance to platelet function.

Last but not least, it has been suggested that PDI act as a modulator of distinct members of Nox enzymes in the vascular system [96]. There is a close association between PDI and Noxl [96-98], phagocytic Nox2 [99, 100], and Nox4 [97, 101], which has been demonstrated through biochemical and molecular approaches of gene silencing and overexpression. Specifically to Nox2, PDI regulates its function possibly 
(a)

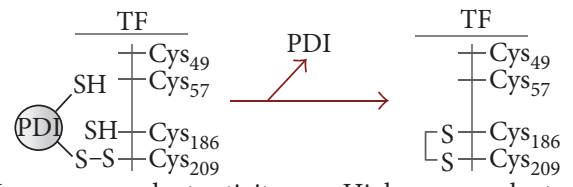

Low procoagulant activity

High procoagulant activity

(b)<smiles>CCCCCCCC</smiles>

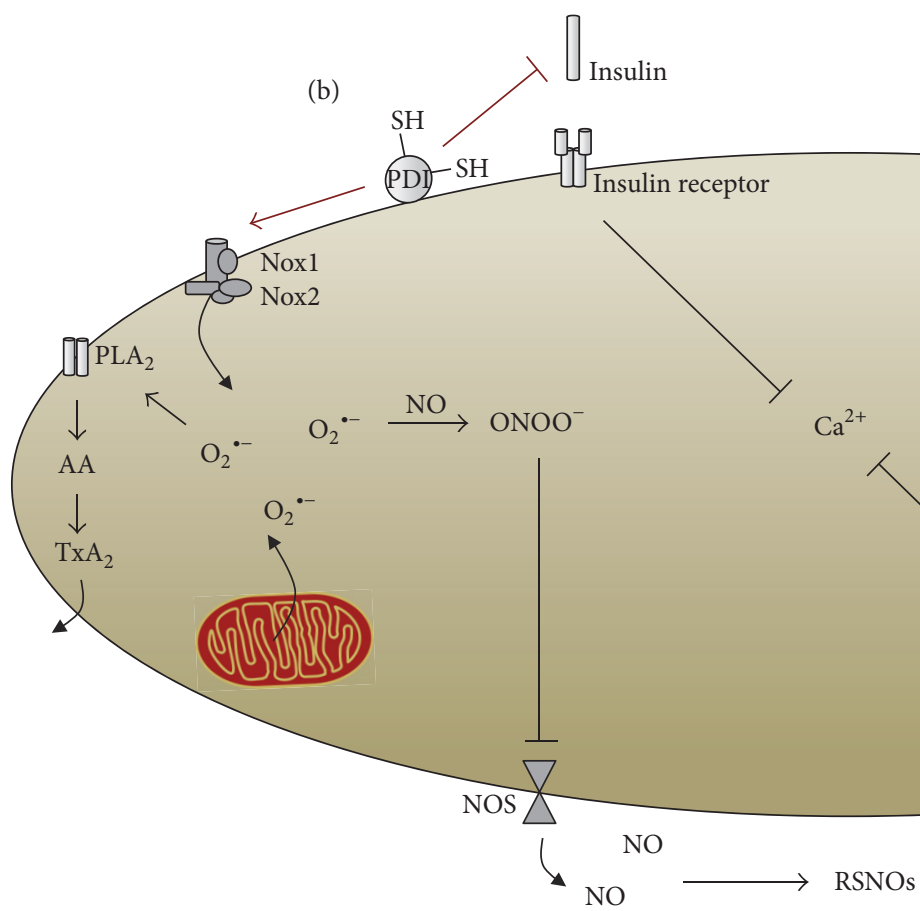

(c)

c)

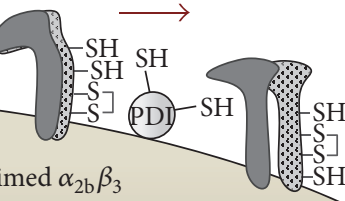
Activated $\alpha_{2 \mathrm{~b}} \beta_{3}$
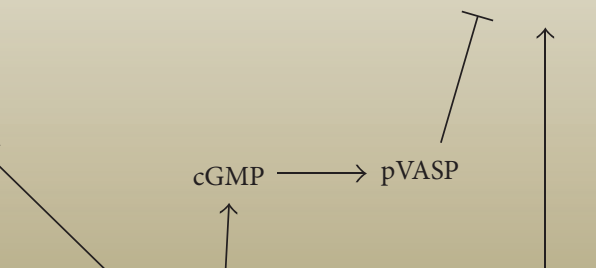

$\mathrm{O}$

(d)

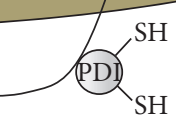

FIGURE 2: PDI participates in mechanisms of platelet hyperactivation induced by metabolic syndrome. In (a), PDI promotes the procoagulant activity of tissue factor (TF) through the formation of a disulfide bond between TF Cys ${ }_{186}$ and $\mathrm{Cys}_{209}$. In (b), PDI inhibits insulin's action by reducing a disulfide bond that precipitates insulin's $\beta$-chain, preventing insulin's inhibitory activity upon TF and insulin's intracellular signaling in platelets. Also, PDI regulates Nox enzymes, promoting stronger generation of $\mathrm{O}_{2}{ }^{--}$that can either react with NO, forming peroxynitrite that will inhibit nitric oxide synthase (NOS) or induce thromboxane (e.g., $\mathrm{A}_{2}$ ) generation through phospholipase $\mathrm{A}_{2}\left(\mathrm{PLA} \mathrm{A}_{2}\right.$ ) and subsequent COX-derived arachidonic acid (AA) platelet metabolism. (c) PDI promotes the isomerization of a disulfide bond in $\alpha_{2 \mathrm{~b}} \beta_{3}$ integrin. Finally, in (d), PDI has a paradoxical effect in platelet aggregation, acting as a nitric oxide (NO) carrier and releaser through transnitrosation and denitrosation reactions of S-nitrosothiols (RSNOs). Arrows in red indicate overactivated mechanisms.

through mechanisms involving thiol groups on its various subunits and therefore contribute to ROS generation [96]. Early studies have found increased protein expression and activity of Nox 2 subunits $\mathrm{p} 22^{\text {phox }}$ and gp91 ${ }^{\text {phox }}$ in pMPs from septic patients [102], which were later demonstrated also to present higher levels of PDI, as well [103]. Even though there is no study addressing such interaction in platelets from MetS patients, it is plausible to infer that PDI might also regulate Nox 2 activity in these cells, further contributing for OxSdriven NOS uncoupling, thromboxane generation as well as insulin resistance.

\section{Conclusions}

MetS increases cardiovascular risk and mortality, being considered a worldwide epidemic. Among the cardiovascular outcomes implicated in MetS, platelet hyperactivation plays a pivotal role in morbidity and mortality. At the same time, PDI is an important regulator of platelet function. However, to the best of our knowledge, no study has investigated the likely contribution of PDI in MetS-induced platelet hyperactivity, nor has it ever been proposed. Therefore, we propose that PDI could be a potential culprit of MetSinduced platelet hyperactivity, possibly through a deficient PDI denitrosation activity, decreased PDI S-nitrosylation and/or less PDI needed for transnitrosation reactions, an increase in TF activation, and insulin resistance caused by increased quantity and/or activity of secreted platelet PDI. Novel original studies are needed to corroborate or reject this hypothesis.

\section{Competing Interests}

The authors declare no actual or potential conflict of interests. 


\section{Acknowledgments}

The authors thank the Fundação de Amparo à Pesquisa e ao Desenvolvimento Científico e Tecnológico do Maranhão, FAPEMA (APP 0395/12, ESTAGIO 0814/13, APCINTER 02698/14) and Conselho Nacional de Desenvolvimento Científico e Tecnológico, CNPq (485251/2012-4). Andrés Trostchansky was supported by CSIC grupos I+D 2014 (536) and FAPEMA (PVI-05558/15).

\section{References}

[1] K. G. Alberti, R. H. Eckel, S. M. Grundy et al., "Harmonizing the metabolic syndrome: a joint interim statement of the International Diabetes Federation Task Force on Epidemiology and Prevention; National Heart, Lung, and Blood Institute; American Heart Association; World Heart Federation; International Atherosclerosis Society; and International Association for the Study of Obesity," Circulation, vol. 120, pp. 1640-1645, 2009.

[2] J. Kaur, "A Comprehensive Review on Metabolic Syndrome," Cardiology Research and Practice, vol. 2014, Article ID 943162, 21 pages, 2014.

[3] E. S. Ford, W. H. Giles, and W. H. Dietz, "Prevalence of the metabolic syndrome among US adults: findings from the third National Health and Nutrition Examination Survey," The Journal of the American Medical Association, vol. 287, no. 3, pp. 356-359, 2002.

[4] A. Towfighi and B. Ovbiagele, "Metabolic syndrome and stroke," Current Diabetes Reports, vol. 8, no. 1, pp. 37-41, 2008.

[5] E. Kassi, P. Pervanidou, G. Kaltsas, and G. Chrousos, "Metabolic syndrome: definitions and controversies," BMC Medicine, vol. 9, article 48, 2011.

[6] M.-J. van Rooy, W. Duim, R. Ehlers, A. V. Buys, and E. Pretorius, "Platelet hyperactivity and fibrin clot structure in transient ischemic attack individuals in the presence of metabolic syndrome: a microscopy and thromboelastography ${ }^{\circledR}$ study," Cardiovascular Diabetology, vol. 14, article 86, 2015.

[7] M.-J. Van Rooy and E. Pretorius, "Metabolic syndrome, platelet activation and the development of transient ischemic attack or thromboembolic stroke," Thrombosis Research, vol. 135, no. 3, pp. 434-442, 2015.

[8] M. Mathew, E. Tay, and K. Cusi, "Elevated plasma free fatty acids increase cardiovascular risk by inducing plasma biomarkers of endothelial activation, myeloperoxidase and PAI-1 in healthy subjects," Cardiovascular Diabetology, vol. 9, article 9, 2010.

[9] L. K. Jennings, "Mechanisms of platelet activation: need for new strategies to protect against platelet-mediated atherothrombosis," Thrombosis and Haemostasis, vol. 102, no. 2, pp. 248-257, 2009.

[10] F. Santilli, D. Lapenna, S. La Barba, and G. Davì, "Oxidative stress-related mechanisms affecting response to aspirin in diabetes mellitus," Free Radical Biology and Medicine, vol. 80, pp. 101-110, 2015.

[11] L. Vizioli, S. Muscari, and A. Muscari, “The relationship of mean platelet volume with the risk and prognosis of cardiovascular diseases," International Journal of Clinical Practice, vol. 63, no. 10, pp. 1509-1515, 2009.

[12] F. Santilli, N. Vazzana, R. Liani, M. T. Guagnano, and G. Davì, "Platelet activation in obesity and metabolic syndrome," Obesity Reviews, vol. 13, no. 1, pp. 27-42, 2012.
[13] S.-W. Wan, C.-F. Lin, Y.-T. Lu, H.-Y. Lei, R. Anderson, and Y.S. Lin, "Endothelial cell surface expression of protein disulfide isomerase activates $\beta 1$ and $\beta 3$ integrins and facilitates dengue virus infection," Journal of Cellular Biochemistry, vol. 113, no. 5, pp. 1681-1691, 2012.

[14] K. Chen, Y. Lin, and T. C. Detwiler, "Protein disulfide isomerase activity is released by activated platelets," Blood, vol. 79, no. 9, pp. 2226-2228, 1992.

[15] C. Wang, W. Li, J. Ren et al., "Structural insights into the redoxregulated dynamic conformations of human protein disulfide isomerase," Antioxidants \& Redox Signaling, vol. 19, no. 1, pp. 36-45, 2013.

[16] M. Schwaller, B. Wilkinson, and H. F. Gilbert, "Reductionreoxidation cycles contribute to catalysis of disulfide isomerization by protein-disulfide isomerase," The Journal of Biological Chemistry, vol. 278, no. 9, pp. 7154-7159, 2003.

[17] J. Cho, D. R. Kennedy, L. Lin et al., "Protein disulfide isomerase capture during thrombus formation in vivo depends on the presence of $\beta 3$ integrins," Blood, vol. 120, no. 3, pp. 647-655, 2012.

[18] D. W. Essex, "Redox control of platelet function," Antioxidants \& Redox Signaling, vol. 11, no. 5, pp. 1191-1225, 2009.

[19] J. Lahav, E. M. Wijnen, O. Hess et al., "Enzymatically catalyzed disulfide exchange is required for platelet adhesion to collagen via integrin $\alpha_{2} \beta_{1}$," Blood, vol. 102, no. 6, pp. 2085-2092, 2003.

[20] J. K. Burgess, K. A. Hotchkiss, C. Suter et al., "Physical proximity and functional association of glycoprotein $1 \mathrm{~b} \alpha$ and proteindisulfide isomerase on the platelet plasma membrane," The Journal of Biological Chemistry, vol. 275, no. 13, pp. 9758-9766, 2000.

[21] D. W. Essex, A. Miller, M. Swiatkowska, and R. D. Feinman, "Protein disulfide isomerase catalyzes the formation of disulfide-linked complexes of vitronectin with thrombinantithrombin," Biochemistry, vol. 38, no. 32, pp. 10398-10405, 1999.

[22] K. A. Hotchkiss, C. N. Chesterman, and P. J. Hogg, "Catalysis of disulfide isomerization in thrombospondin 1 by protein disulfide isomerase," Biochemistry, vol. 35, no. 30, pp. 9761-9767, 1996.

[23] V. Darley-Usmar and B. Halliwell, "Blood radicals: reactive nitrogen species, reactive oxygen species, transition metal ions, and the vascular system," Pharmaceutical Research, vol. 13, no. 5, pp. 649-662, 1996.

[24] K. K. Griendling, "NADPH oxidases: new regulators of old functions," Antioxidants and Redox Signaling, vol. 8, no. 9-10, pp. 1443-1445, 2006.

[25] D. P. Jones, "Redefining oxidative stress," Antioxidants and Redox Signaling, vol. 8, no. 9-10, pp. 1865-1879, 2006.

[26] C. K. Roberts and K. K. Sindhu, "Oxidative stress and metabolic syndrome," Life Sciences, vol. 84, no. 21-22, pp. 705-712, 2009.

[27] D. P. Jones and Y.-M. Go, "Redox compartmentalization and cellular stress," Diabetes, Obesity and Metabolism, vol. 12, supplement 2, pp. 116-125, 2010.

[28] Y.-M. Go and D. P. Jones, "Cysteine/cystine redox signaling in cardiovascular disease," Free Radical Biology and Medicine, vol. 50, no. 4, pp. 495-509, 2011.

[29] Y. Spanidis, A. Mpesios, D. Stagos et al., "Assessment of the redox status in patients with metabolic syndrome and type 2 diabetes reveals great variations," Experimental and Therapeutic Medicine, vol. 11, no. 3, pp. 895-903, 2016. 
[30] L. J. da Fonseca, V. Nunes-Souza, S. Guedes Gda, G. SchettinoSilva, M. A. Mota-Gomes, and L. A. Rabelo, "Oxidative status imbalance in patients with metabolic syndrome: role of the myeloperoxidase/hydrogen peroxide axis," Oxidative Medicine and Cellular Longevity, vol. 2014, Article ID 898501, 14 pages, 2014.

[31] B. Lassègue and K. K. Griendling, "NADPH oxidases: functions and pathologies in the vasculature," Arteriosclerosis, Thrombosis, and Vascular Biology, vol. 30, no. 4, pp. 653-661, 2010.

[32] B. Lassègue, A. San Martín, and K. K. Griendling, "Biochemistry, physiology, and pathophysiology of NADPH oxidases in the cardiovascular system," Circulation Research, vol. 110, no. 10, pp. 1364-1390, 2012.

[33] J. D. Lambeth, T. Kawahara, and B. Diebold, "Regulation of Nox and Duox enzymatic activity and expression," Free Radical Biology and Medicine, vol. 43, no. 3, pp. 319-331, 2007.

[34] R. E. Clempus, D. Sorescu, A. E. Dikalova et al., "Nox4 is required for maintenance of the differentiated vascular smooth muscle cell phenotype," Arteriosclerosis, Thrombosis, and Vascular Biology, vol. 27, no. 1, pp. 42-48, 2007.

[35] M. Y. Lee, A. San Martin, P. K. Mehta et al., "Mechanisms of vascular smooth muscle NADPH oxidase 1 (Noxl) contribution to injury-induced neointimal formation," Arteriosclerosis, Thrombosis, and Vascular Biology, vol. 29, no. 4, pp. 480-487, 2009.

[36] K. D. Martyn, L. M. Frederick, K. Von Loehneysen, M. C. Dinauer, and U. G. Knaus, "Functional analysis of Nox4 reveals unique characteristics compared to other NADPH oxidases," Cellular Signalling, vol. 18, no. 1, pp. 69-82, 2006.

[37] J. D. Van Buul, M. Fernandez-Borja, E. C. Anthony, and P. L. Hordijk, "Expression and localization of NOX2 and NOX4 in primary human endothelial cells," Antioxidants and Redox Signaling, vol. 7, no. 3-4, pp. 308-317, 2005.

[38] G. R. Drummond and C. G. Sobey, "Endothelial NADPH oxidases: which NOX to target in vascular disease?" Trends in Endocrinology and Metabolism, vol. 25, no. 9, pp. 452-463, 2014.

[39] P. Pignatelli, R. Carnevale, S. Di Santo et al., "Inherited Human gp9lphox deficiency is associated with impaired isoprostane formation and platelet dysfunction," Arteriosclerosis, Thrombosis, and Vascular Biology, vol. 31, no. 2, pp. 423-434, 2011.

[40] T. Seno, N. Inoue, D. Gao et al., "Involvement of NADH/NADPH oxidase in human platelet ROS production," Thrombosis Research, vol. 103, no. 5, pp. 399-409, 2001.

[41] D. Vara, M. Campanella, and G. Pula, "The novel NOX inhibitor 2-acetylphenothiazine impairs collagen-dependent thrombus formation in a GPVI-dependent manner," British Journal of Pharmacology, vol. 168, no. 1, pp. 212-224, 2013.

[42] M. K. Delaney, K. Kim, B. Estevez et al., "Differential roles of the NADPH-oxidase 1 and 2 in platelet activation and thrombosis," Arteriosclerosis, Thrombosis, and Vascular Biology, vol. 36, no. 5, pp. 846-854, 2016.

[43] C. A. Papaharalambus and K. K. Griendling, "Basic mechanisms of oxidative stress and reactive oxygen species in cardiovascular injury," Trends in Cardiovascular Medicine, vol. 17, no. 2, pp. 48-54, 2007.

[44] M. Kitada, Z. Zhang, A. Mima, and G. L. King, "Molecular mechanisms of diabetic vascular complications," Journal of Diabetes Investigation, vol. 1, no. 3, pp. 77-89, 2010.

[45] M. Brownlee, "Biochemistry and molecular cell biology of diabetic complications," Nature, vol. 414, no. 6865, pp. 813-820, 2001.
[46] R. P. Robertson, J. Harmon, P. O. T. Tran, and V. Poitout, “ $\beta$-Cell glucose toxicity, lipotoxicity, and chronic oxidative stress in type 2 diabetes," Diabetes, vol. 53, supplement 1, pp. S119-S124, 2004.

[47] D. C. L. Masquio, A. De Piano, R. M. S. Campos et al., "The role of multicomponent therapy in the metabolic syndrome, inflammation and cardiovascular risk in obese adolescents," British Journal of Nutrition, vol. 113, no. 12, pp. 1920-1930, 2015.

[48] G. Anfossi, I. Russo, and M. Trovati, "Resistance to aspirin and thienopyridines in diabetes mellitus and metabolic syndrome," Current Vascular Pharmacology, vol. 6, no. 4, pp. 313-328, 2008.

[49] S. Schildknecht, B. van der Loo, K. Weber, K. Tiefenthaler, A. Daiber, and M. M. Bachschmid, "Endogenous peroxynitrite modulates PGHS-1-dependent thromboxane A2 formation and aggregation in human platelets," Free Radical Biology and Medicine, vol. 45, no. 4, pp. 512-520, 2008.

[50] C. Patrono, "The Multifaceted clinical readouts of platelet inhibition by low-dose aspirin," Journal of the American College of Cardiology, vol. 66, no. 1, pp. 74-85, 2015.

[51] L. P. Audoly, B. Rocca, J. E. Fabre et al., "Cardiovascular responses to the isoprostanes $\mathrm{iPF}(2 \alpha)$-III and $\mathrm{iPE}(2)$-III are mediated via the thromboxane $\mathrm{A}(2)$ receptor in vivo," Circulation, vol. 101, no. 24, pp. 2833-2840, 2000.

[52] C. Patrono, A. Falco, and G. Davì, "Isoprostane formation and inhibition in atherothrombosis," Current Opinion in Pharmacology, vol. 5, no. 2, pp. 198-203, 2005.

[53] G. Davì, M. T. Guagnano, G. Ciabattoni et al., "Platelet activation in obese women: role of inflammation and oxidant stress," Journal of the American Medical Association, vol. 288, no. 16, pp. 2008-2014, 2002.

[54] S. Magwenzi, C. Woodward, K. S. Wraith et al., "Oxidized LDL activates blood platelets through CD36/NOX2-mediated inhibition of the cGMP/protein kinase G signaling cascade," Blood, vol. 125, no. 17, pp. 2693-2703, 2015.

[55] A. Trostchansky, V. B. O’Donnell, D. C. Goodwin et al., "Interactions between nitric oxide and peroxynitrite during prostaglandin endoperoxide $\mathrm{H}$ synthase-1 catalysis: a free radical mechanism of inactivation," Free Radical Biology and Medicine, vol. 42, no. 7, pp. 1029-1038, 2007.

[56] N. P. Andrews, M. Husain, N. Dakak, and A. A. Quyyumi, "Platelet inhibitory effect of nitric oxide in the human coronary circulation: impact of endothelial dysfunction," Journal of the American College of Cardiology, vol. 37, no. 2, pp. 510-516, 2001.

[57] J. C. de Graaf, J. D. Banga, S. Moncada, R. M. J. Palmer, P. G. de Groot, and J. J. Sixma, "Nitric oxide functions as an inhibitor of platelet adhesion under flow conditions," Circulation, vol. 85, no. 6, pp. 2284-2290, 1992.

[58] U. Hink, H. Li, H. Mollnau et al., "Mechanisms underlying endothelial dysfunction in diabetes mellitus," Circulation research, vol. 88, no. 2, pp. E14-E22, 2001.

[59] A. Lerman and A. M. Zeiher, "Endothelial function: cardiac events," Circulation, vol. 111, no. 3, pp. 363-368, 2005.

[60] I. Russo, G. Doronzo, L. Mattiello, A. De Salve, M. Trovati, and G. Anfossi, "The activity of constitutive nitric oxide synthase is increased by the pathway cAMP/cAMP-activated protein kinase in human platelets. New insights into the antiaggregating effects of cAMP-elevating agents," Thrombosis Research, vol. 114, no. 4, pp. 265-273, 2004.

[61] E. Fuentes and I. Palomo, "Role of oxidative stress on platelet hyperreactivity during aging," Life Sciences, vol. 148, pp. 17-23, 2016. 
[62] H. W. Kwon, J. H. Shin, H. J. Cho, M. H. Rhee, and H. J. Park, "Total saponin from Korean Red Ginseng inhibits binding of adhesive proteins to glycoprotein IIb/IIIa via phosphorylation of VASP (Ser(157)) and dephosphorylation of PI3K and Akt," Journal of Ginseng Research, vol. 40, pp. 76-85, 2016.

[63] D. Banerjee, S. Mazumder, and A. Kumar Sinha, "Involvement of nitric oxide on calcium mobilization and arachidonic acid pathway activation during platelet aggregation with different aggregating agonists," International Journal of Biomedical Science, vol. 12, pp. 25-35, 2016.

[64] G.-R. Wang, Y. Zhu, P. V. Halushka, T. M. Lincoln, and M. E. Mendelsohn, "Mechanism of platelet inhibition by nitric oxide: In vivo phosphorylation of thromboxane receptor by cyclic GMP-dependent protein kinase," Proceedings of the National Academy of Sciences of the United States of America, vol. 95, no. 9, pp. 4888-4893, 1998.

[65] J. E. Freedman, J. Loscalzo, M. R. Barnard, C. Alpert, J. F. Keaney Jr., and A. D. Michelson, "Nitric oxide released from activated platelets inhibits platelet recruitment," The Journal of Clinical Investigation, vol. 100, no. 2, pp. 350-356, 1997.

[66] L. A. Blatter and W. G. Wier, "Nitric oxide decreases $\left[\mathrm{Ca}^{2+}\right]_{i}$ in vascular smooth muscle by inhibition of the calcium current," Cell Calcium, vol. 15, no. 2, pp. 122-131, 1994.

[67] P. F. Monteiro, R. P. Morganti, M. A. Delbin et al., "Platelet hyperaggregability in high-fat fed rats: a role for intraplatelet reactive-oxygen species production," Cardiovascular Diabetology, vol. 11, article 5, 2012.

[68] A. S. Brown, M. A. Moro, J. M. Masse, E. M. Cramer, M. Radomski, and V. Darley-Usmar, "Nitric oxide-dependent and independent effects on human platelets treated with peroxynitrite," Cardiovascular Research, vol. 40, no. 2, pp. 380-388, 1998.

[69] F. Paneni, J. A. Beckman, M. A. Creager, and F. Cosentino, "Diabetes and vascular disease: pathophysiology, clinical consequences, and medical therapy: part I," European Heart Journal, vol. 34, no. 31, pp. 2436-2443, 2013.

[70] T. E. Suslova, A. V. Sitozhevskii, O. N. Ogurkova et al., "Platelet hemostasis in patients with metabolic syndrome and type 2 diabetes mellitus: cGMP- and NO-dependent mechanisms in the insulin-mediated platelet aggregation," Frontiers in Physiology, vol. 6, article 501, 2015.

[71] R. A. Anderson, G. R. Ellis, Y. Y. Chirkov et al., "Determinants of platelet responsiveness to nitric oxide in patients with chronic heart failure," European Journal of Heart Failure, vol. 6, no. 1, pp. 47-54, 2004.

[72] A. S. Hajek, J. H. Joist, R. K. Baker, L. Jarett, and W. H. Daughaday, "Demonstration and partial characterization of insulin receptors in human platelets," The Journal of Clinical Investigation, vol. 63, no. 5, pp. 1060-1065, 1979.

[73] C. Falcon, G. Pfliegler, H. Deckmyn, and J. Vermylen, “The platelet insulin receptor: detection, partial characterization, and search for a function," Biochemical and Biophysical Research Communications, vol. 157, no. 3, pp. 1190-1196, 1988.

[74] M. Trovati, E. M. Mularoni, S. Burzacca et al., "Impaired insulininduced platelet antiaggregating effect in obesity and in obese NIDDM patients," Diabetes, vol. 44, no. 11, pp. 1318-1322, 1995.

[75] M. Udvardy, G. Pfliegler, and K. Rak, "Platelet insulin receptor determination in non-insulin dependent diabetes mellitus," Experientia, vol. 41, no. 3, pp. 422-423, 1985.

[76] R. M. Touyz and E. L. Schiffrin, "Blunted inhibition by insulin of agonist-stimulated calcium, ph and aggregatory responses in platelets from hypertensive patients," Journal of Hypertension, vol. 12, no. 11, pp. 1255-1263, 1994.
[77] M. Trovati, G. Anfossi, F. Cavalot, P. Massucco, E. Mularoni, and G. Emanuelli, "Insulin directly reduces platelet sensitivity to aggregating agents. Studies in vitro and in vivo," Diabetes, vol. 37, no. 6, pp. 780-786, 1988.

[78] M. Trovati, G. Anfossi, P. Massucco et al., "Insulin stimulates nitric oxide synthesis in human platelets and, through nitric oxide, increases platelet concentrations of both guanosine- 3,5'cyclic monophosphate and adenosine-3,5'-cyclic monophosphate," Diabetes, vol. 46, no. 5, pp. 742-749, 1997.

[79] A. I. Vinik, T. Erbas, T. Sun Park, R. Nolan, and G. L. Pittenger, "Platelet dysfunction in type 2 diabetes," Diabetes Care, vol. 24, no. 8, pp. 1476-1485, 2001.

[80] M. Konieczynska, K. Fil, M. Bazanek, and A. Undas, "Prolonged duration of type 2 diabetes is associated with increased thrombin generation, prothrombotic fibrin clot phenotype and impaired fibrinolysis," Thrombosis and Haemostasis, vol. 111, no. 4, pp. 685-693, 2014.

[81] M. Crescente, F. G. Pluthero, L. Li et al., "Intracellular trafficking, localization, and mobilization of platelet-borne thiol isomerases," Arteriosclerosis, Thrombosis, and Vascular Biology, vol. 36, no. 6, pp. 1164-1173, 2016.

[82] D. W. Essex, K. Chen, and M. Swiatkowska, "Localization of protein disulfide isomerase to the external surface of the platelet plasma membrane," Blood, vol. 86, no. 6, pp. 2168-2173, 1995.

[83] J. Zhou, Y. Wu, L. Wang et al., "The C-terminal CGHC motif of protein disulfide isomerase supports thrombosis," The Journal of Clinical Investigation, vol. 125, no. 12, pp. 4391-4406, 2015.

[84] C. Reinhardt, M.-L. Von Brühl, D. Manukyan et al., "Protein disulfide isomerase acts as an injury response signal that enhances fibrin generation via tissue factor activation," Journal of Clinical Investigation, vol. 118, no. 3, pp. 1110-1122, 2008.

[85] A. Holmgren, "Thioredoxin catalyzes the reduction of insulin disulfides by dithiothreitol and dihydrolipoamide," The Journal of Biological Chemistry, vol. 254, no. 19, pp. 9627-9632, 1979.

[86] G. Tsimerman, A. Roguin, A. Bachar, E. Melamed, B. Brenner, and A. Aharon, "Involvement of microparticles in diabetic vascular complications," Thrombosis and Haemostasis, vol. 106, no. 2, pp. 310-321, 2011.

[87] A. Raturi, S. Miersch, J. W. Hudson, and B. Mutus, "Platelet microparticle-associated protein disulfide isomerase promotes platelet aggregation and inactivates insulin," Biochimica et Biophysica Acta (BBA)_Biomembranes, vol. 1778, no. 12, pp. 2790-2796, 2008.

[88] J. Chiu, F. Passam, D. Butera, and P. J. Hogg, "Protein disulfide isomerase in thrombosis," Seminars in Thrombosis and Hemostasis, vol. 41, no. 7, pp. 765-773, 2015.

[89] N. Ramachandran, P. Root, X.-M. Jiang, P. J. Hogg, and B. Mutus, "Mechanism of transfer of NO from extracellular Snitrosothiols into the cytosol by cell-surface protein disulfide isomerase," Proceedings of the National Academy of Sciences of the United States of America, vol. 98, no. 17, pp. 9539-9544, 2001.

[90] A. Zai, M. A. Rudd, A. W. Scribner, and J. Loscalzo, "Cellsurface protein disulfide isomerase catalyzes transnitrosation and regulates intracellular transfer of nitric oxide," Journal of Clinical Investigation, vol. 103, no. 3, pp. 393-399, 1999.

[91] P. Root, I. Sliskovic, and B. Mutus, "Platelet cell-surface protein disulphide-isomerase mediated S-nitrosoglutathione consumption," Biochemical Journal, vol. 382, no. 2, pp. 575-580, 2004.

[92] S. E. Bell, C. M. Shah, and M. P. Gordge, "Protein disulfideisomerase mediates delivery of nitric oxide redox derivatives into platelets," Biochemical Journal, vol. 403, no. 2, pp. 283-288, 2007. 
[93] T. Uehara, T. Nakamura, D. Yao et al., "S-Nitrosylated proteindisulphide isomerase links protein misfolding to neurodegeneration," Nature, vol. 441, no. 7092, pp. 513-517, 2006.

[94] E. J. Heckler, V. Kholodovych, M. Jain, T. Liu, H. Li, and A. Beuve, "Mapping soluble guanylyl cyclase and protein disulfide isomerase regions of interaction," PLoS ONE, vol. 10, no. 11, Article ID e0143523, 2015.

[95] E. J. Heckler, P.-A. Crassous, P. Baskaran, and A. Beuve, "Protein disulfide-isomerase interacts with soluble guanylyl cyclase via a redox-based mechanism and modulates its activity;" Biochemical Journal, vol. 452, no. 1, pp. 161-169, 2013.

[96] F. R. M. Laurindo, D. C. Fernandes, A. M. Amanso, L. R. Lopes, and C.X. C. Santos, "Novel role of protein disulfide isomerase in the regulation of NADPH oxidase activity: pathophysiological implications in vascular diseases," Antioxidants and Redox Signaling, vol. 10, no. 6, pp. 1101-1113, 2008.

[97] D. C. Fernandes, A. H. O. Manoel, J. Wosniak Jr., and F. R. Laurindo, "Protein disulfide isomerase overexpression in vascular smooth muscle cells induces spontaneous preemptive NADPH oxidase activation and Noxl mRNA expression: effects of nitrosothiol exposure," Archives of Biochemistry and Biophysics, vol. 484, no. 2, pp. 197-204, 2009.

[98] L. A. Pescatore, D. Bonatto, F. L. Forti, A. Sadok, H. Kovacic, and F. R. M. Laurindo, "Protein disulfide isomerase is required for platelet-derived growth factor-induced vascular smooth muscle cell migration, Noxl NADPH oxidase expression, and RhoGTPase activation," The Journal of Biological Chemistry, vol. 287, no. 35, pp. 29290-29300, 2012.

[99] A. M. A. de Paes, S. Veríssimo-Filho, L. L. Guimarães et al., "Protein disulfide isomerase redoxdependent association with $\mathrm{p} 47^{\text {phox }}$ : evidence for an organizer role in leukocyte NADPH oxidase activation," Journal of Leukocyte Biology, vol. 90, no. 4, pp. 799-810, 2011.

[100] C. X. C. Santos, B. S. Stolf, P. V. A. Takemoto et al., "Protein disulfide isomerase (PDI) associates with NADPH oxidase and is required for phagocytosis of Leishmania chagasi promastigotes by macrophages," Journal of Leukocyte Biology, vol. 86, no. 4, pp. 989-998, 2009.

[101] M. Janiszewski, L. R. Lopes, A. O. Carmo et al., "Regulation of $\mathrm{NAD}(\mathrm{P}) \mathrm{H}$ oxidase by associated protein disulfide isomerase in vascular smooth muscle cells," Journal of Biological Chemistry, vol. 280, no. 49, pp. 40813-40819, 2005.

[102] M. Janiszewski, A. O. Do Carmo, M. A. Pedro, E. Silva, E. Knobel, and F. R. M. Laurindo, "Platelet-derived exosomes of septic individuals possess proapoptotic NAD(P)H oxidase activity: a novel vascular redox pathway," Critical Care Medicine, vol. 32, no. 3, pp. 818-825, 2004.

[103] M. H. Gambim, A. de Oliveira do Carmo, L. Marti, S. VeríssimoFilho, L. R. Lopes, and M. Janiszewski, "Platelet-derived exosomes induce endothelial cell apoptosis through peroxynitrite generation: experimental evidence for a novel mechanism of septic vascular dysfunction," Critical Care, vol. 11 article R107, 2007. 


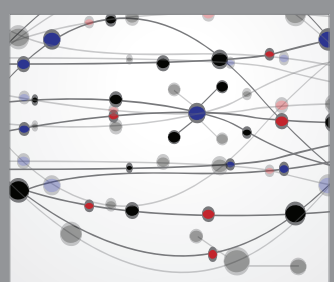

The Scientific World Journal
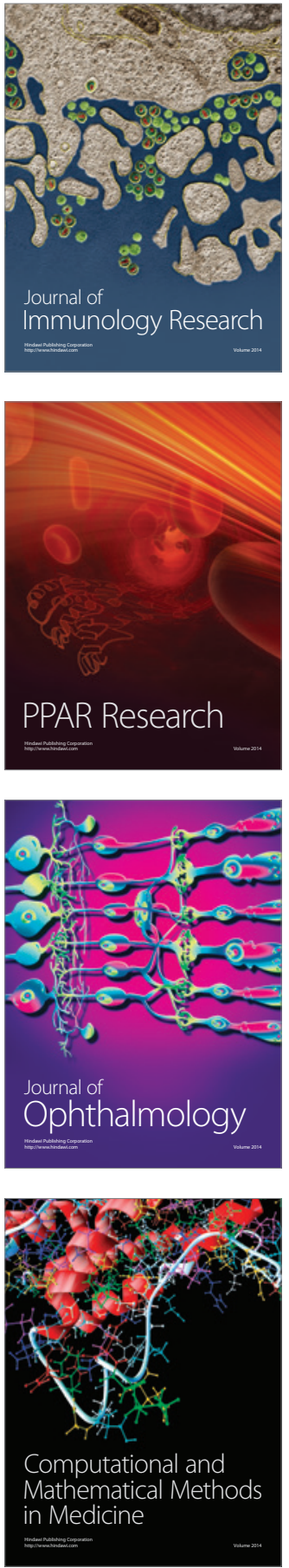

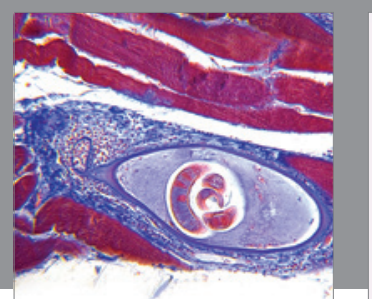

Gastroenterology Research and Practice

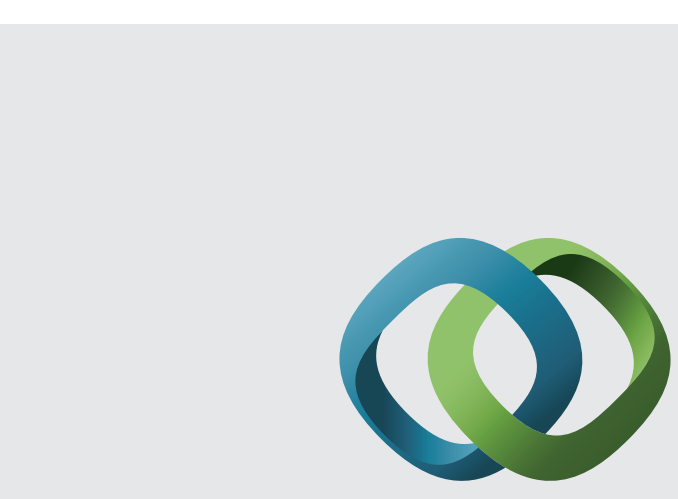

\section{Hindawi}

Submit your manuscripts at

http://www.hindawi.com
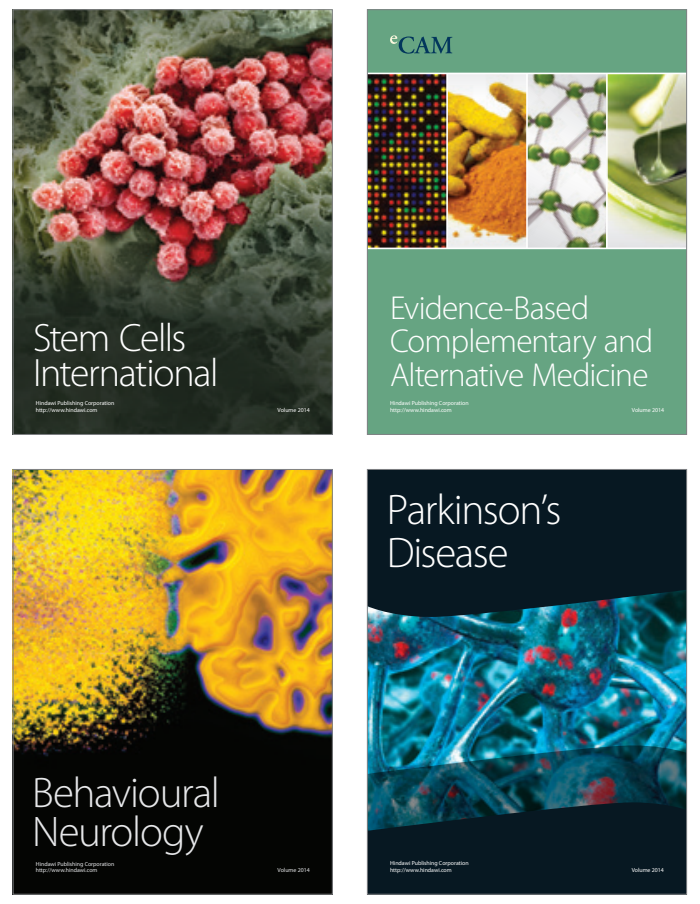
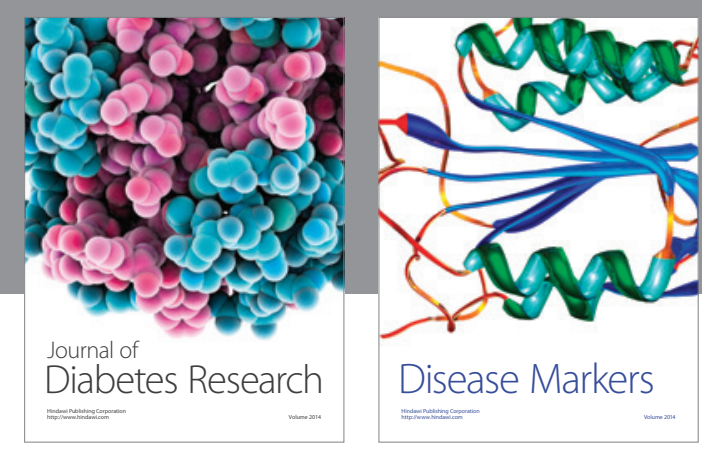

Disease Markers
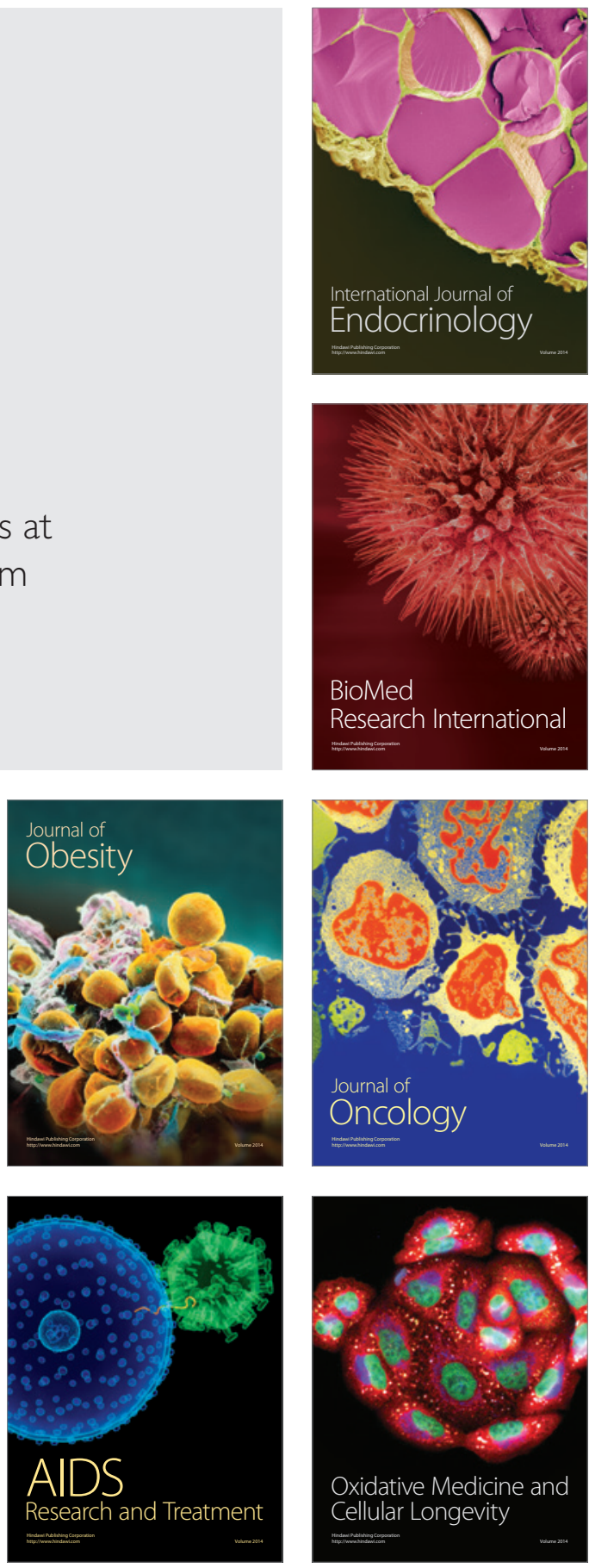